\title{
Blinkers in the solar transition region
}

\author{
J.M.A. Ashbourn ${ }^{1, a}$, L.C. Woods ${ }^{1}$, and D.T. Kennedy ${ }^{2}$ \\ 1 Mathematical Institute, University of Oxford, 24-29 St Giles', Oxford OX1 3LB, UK \\ 2 Merton College, University of Oxford, Merton Street, Oxford OX1 4JD, UK
}

Received 7 September 2016 / Received in final form 27 November 2016

Published online 2 March 2017

(C) The Author(s) 2017. This article is published with open access at Springerlink.com

\begin{abstract}
In this paper it is shown that the small flare-like patches known as 'blinkers' may be explained as the result of an instability in transition layer magnetic flux tubes, which are represented by a set of isolated, high-aspect ratio vertical cylinders as the legs of coronal plasma loops. An essential prerequisite for the instability is that the heating, which is assumed to be only due to ohmic dissipation, closely balances the radiation losses. The instability occurs in the plasma pressure and could be triggered by a random shock wave; it rapidly increases the local pressure and results in a minor 'explosion'. By dissipating energy away from the region, this reduces the local pressure and restores stability on a timescale of a few minutes, which is in agreement with observations of blinkers. The theory is an extension of Ashbourn and Woods' previous treatment of the transition layer differential emission measure.
\end{abstract}

\section{Introduction}

Harrison [1] identified small flare-like patches that suddenly brighten at transition region temperatures, which he called "blinkers". The precise nature of blinkers in the solar transition region is of longstanding interest in the scientific community and has garnered significant attention in the literature. However, despite a number of studies on this observed phenomenon, there are still many unanswered questions on blinkers and the mechanisms which drive them. In this paper we present a mathematical model of the transition layer magnetic flux tubes and use this to show that blinkers may be explained as the result of an instability in these magnetic flux tubes.

The variable nature of UV and EUV emission from the solar transition region is well known with the line emission continually changing at every point in the network (i.e. the emission lines vary continually at each point in the observational grid). Nishikawa [2] observed flux enhancements in emission lines at the network boundaries and attributed these to thermal instabilities resulting from the draining of transition material, which Pneuman and Kopp [3] took to be the dominant heat source for the transition region; they found average downward velocities of about $10 \mathrm{~km} \mathrm{~s}^{-1}$ as an estimate for blinker velocities. More recently, Bewsher et al. [4] found the velocities of blinkers to be specifically in the range of $15-25 \mathrm{~km} \mathrm{~s}^{-1}$. It was shown by Harrison [1] that the brightenings increase the intensity of radiation by a factor of about 2.5 over an area $\sim 6000 \times 6000 \mathrm{~km}^{2}$ and persist for an average time of $13 \mathrm{~min}$. Since then a number of careful observations have been made, e.g. by Tarbell

a e-mail: jmaa@seh.ox.ac.uk et al. [5], Subramanian et al. [6], Harrison et al. [1] and Chae et al. [7]. These authors all studied the lifetime of blinkers using a variety of models and observational data and all report similar findings on the lifetime of blinkers. A study by Parnell et al. [8] investigated the lifetime of active region blinkers and showed that these events also persist for an average lifetime of 16-19 min and have many similar properties to quiet region blinkers. In [7] it was found that the patches comprise many small-scale, short-lived separate events, each with a lifetime of between two to three minutes, which were termed "unit brightening events".

The main cause of brightenings is an increase in the emission measure (Krucker et al. [9]) with the observed temperature changing only slightly. One interpretation of this is that the plasma density in the transition layer and lower corona is momentarily enhanced by chromospheric evaporation. Another is that shock waves propagating upwards from the chromosphere increase the local density sufficiently to generate the small-scale events noted above.

The magnetic structure of the transition layer remains uncertain due to resolution limitations, but it seems likely to consist largely of the vertical legs of coronal loops, each of which is fragmented into many threads too fine to be resolved. This idea of blinkers as magnetic flux loops has also been investigated using a magnetic topological model by Submaranian et al. [10] who found that blinker groups are largely associated with the formation of loops after magnetic flux emergence. There are two features of coronal loops that we shall assume also apply to their transition layer legs or rather to the threads comprising them, namely that they have almost uniform thickness (Bray et al. [11]), being on average only $13 \%$ thicker at their midpoints than their footpoints (Klimchuck [12]) and that 
they are circular in cross-section. Klimchuck attributes these properties to the possibility that the magnetic flux tubes are twisted with the tubes conducting electric current around their coronal loops.

The heating of coronal loops is a subject that has attracted considerable attention. Bray et al. [11], Wentzel [13] and Zirker [14] reviewed the principal mechanisms proposed to explain the heating, such as the dissipation of magnetohydrodynamical (MHD) waves, electric currents and magnetic reconnection processes. Our present concern with this coronal problem is incidental, but convection of hot plasma down the loops from the corona is a mechanism that could deliver a considerable amount of energy to the transition region. In fact according to Pneuman and Kopp [3], the downward enthalpy flux exceeds the conductive flux by a considerable amount and is the dominant energy source for the transition region. However it is not certain whether the velocities of several kilometres per second that have been deduced from Doppler measurements (Brekke [15]) are of plasma within the threads comprising the loops or simply of plasma falling under gravity in the less dense region external to the threads.

The outline of this study is as follows: in Section 2 we present our mathematical model which uses the assumptions above to describe the magnetic flux tubes forming the legs of the coronal loops. By applying the principle of conservation of energy (Woods [16]) to this model, we derive equations describing how the pressure inside these flux tubes varies as a function of the transition region temperature. We also show that the model results obtained here agree with the modelled and experimental results discussed in the literature. In Section 3 we consider small perturbations to the flux tube pressure in order to examine the linear stability of the system. Finally, in Section 4 we use this linear stability analysis to explore the nature of blinkers.

\section{Energy equation}

The equation governing the conservation of energy in a magnetoplasma is (e.g. see Woods [16])

$\frac{\partial(\varrho u)}{\partial t}+\nabla \cdot(\varrho \mathbf{v} u)=-\mathbf{p} \cdot \nabla \mathbf{v}-\nabla \cdot \mathbf{q}+\mathbf{j} \cdot(\mathbf{E}+\mathbf{v} \times \mathbf{B})-\mathcal{L}$

where $\varrho$ is the plasma energy, $u$ is the specific internal energy of the plasma, $\mathbf{v}$ is the plasma (fluid) velocity, $\mathbf{p}$ is the plasma (fluid) pressure, $\mathbf{j}$ is the current density, $\mathbf{E}$ is the electric field, $\mathbf{B}$ is the magnetic field vector and $\mathcal{L}$ is the loss rate per unit volume due to radiation. Here $\mathbf{q}$ is the flux of heat.

We shall apply this energy equation to a vertical flux tube or "thread" at a distance $z$ above the photosphere (see Fig. 1). In this figure, $w$ represents the velocity of plasma outside the loop falling under gravity. If we let ${ }^{\prime}=\partial / \partial z$ and assuming that the thread has a circular cross-section of radius $R$ and ignoring radial velocities and
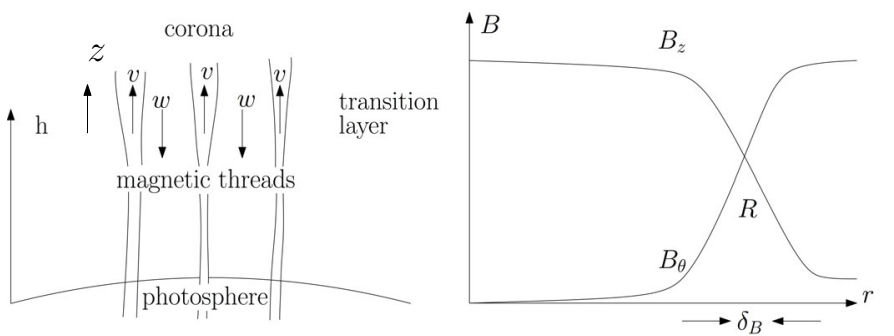

Fig. 1. A schematic showing magnetic threads in the transition layer. The right-hand figure shows how the different components of the magnetic field vary with the radius of the magnetic thread. In the left-hand figure $h$ is the distance above the photosphere and $z$ is the vertical distance inside the magnetic threads.

radial magnetic fields, we obtain from equation (1)

$$
\frac{\partial(\varrho u)}{\partial t}+(\varrho v u)^{\prime}=-q^{\prime}+\mathcal{H}-\mathcal{L}
$$

where $\mathcal{H}$ is the heating rate per unit volume of thread. Here we use our assumption that the only non-zero component of $\mathbf{v}$ is in the $z$-direction and we denote this component $v$. It follows that the derivatives of $v$ only exist in the $z$-direction.

From equation (1) and Ohm's Law, $\eta \mathbf{j}=\mathbf{E}+\mathbf{v} \times \mathbf{B}$, it follows that $\mathcal{H}=\eta j^{2}$, where $\eta$ is the resistivity. The tendency for magnetic fields to steepen rather than diffuse (see Woods [16]) allows us to place the electric current on the edge of the thread as shown in Figure 1, which follows since the components of the magnetic field vary rapidly in this region and hence the current density, which is proportional to these gradients, is much larger here. If this current layer has a thickness $\delta_{B}$ much less than the radius $R$ of the thread, we can adopt the approximation $\mu_{0} j \approx B / \delta_{B}$. Since the ohmic heating rate $\eta j^{2}$ occurs in a volume $2 \pi R L \delta_{B}$, where $R$ is the radius of the flux loop and $L$ is its length, then the equivalent ohmic heating per unit volume of the thread is

$$
\mathcal{H}=2 \eta j^{2} \delta_{B} / R=2 \xi j B / R,
$$

where in standard notation

$$
\xi=\eta / \mu_{0}=\frac{0.51 m_{e}}{\mu_{0} e^{2} n_{e} \tau_{e}}=\frac{5.24 \times 10^{7} \ln \Lambda}{T^{3 / 2}},
$$

is the magnetic diffusivity. Here we have used the standard notation $\mu_{0}$ to denote the permeability of free space, and $e, m_{e}$ and $n_{e}$ represent the charge, mass and number density of an electron respectively. $\tau_{e}$ denotes the collision timescale. The Coulomb logarithm, $\ln \Lambda=16.33+$ $1.5 \ln T-0.5 \ln n_{e}$, is about 15 in the transition region and since it varies quite slowly, we shall adopt this value in the following.

The maximum possible value for the current density, denoted $j_{m}$, will occur when the electrons stream past the ions at the ion sound speed with the speed of the longitudinal waves resulting from the mass of the ions 


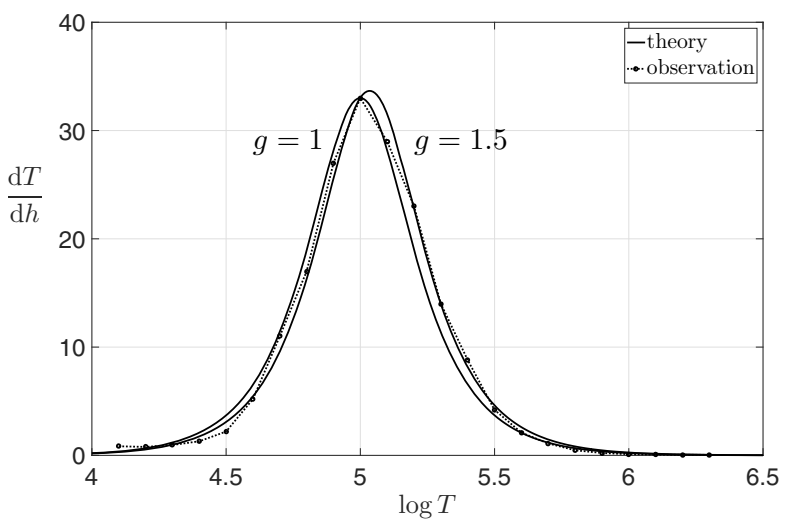

Fig. 2. The temperature gradient $\frac{\mathrm{d} T}{\mathrm{~d} h}\left(\mathrm{~K} \mathrm{~m}^{-1}\right)$ plotted for different temperature values throughout the transition region. The solid lines are the values predicted by the model described in this paper and are calculated using equation (9) with various values of the parameter $g$. The dotted-solid line shows the curve obtained by Mariska [19] using a cell-centred empirical model.

and the pressure of the electrons. When this speed is reached, the ion-acoustic instability sets in (e.g. see Krall and Trivelpiece [17]). Since ohmic heating directly involves the electrons and the rate at which the ions and electrons reach thermal equilibrium is relatively slow, we expect the instability to switch on when $j$ reaches its maximum value of

$$
j_{m}=e n_{e}\left(k_{B} T / m_{i}\right)^{\frac{1}{2}} \approx 1.46 \times 10^{-17} P / T^{\frac{1}{2}} \quad\left(P \equiv n_{e} T\right)
$$

where $m_{i}$ denotes the ion mass and $k_{B}$ is the Boltzmann constant. This instability acts to increase the resistivity, which in turn reduces $j$ and hence causes the instability to switch off. This allows $j$ to again increase up to its maximum and the cycle is repeated. In this way the marginal stability curve is followed, which allows us to adopt equation (5) throughout. Thus from equations (3) to (5) we find

$$
\mathcal{H}=2.3 \times 10^{13} \wp B /\left(T^{2} R\right) \quad\left(\wp \equiv P / 10^{21}\right),
$$

where observations show that on average $\wp$ is a number of the order of unity.

In Ashbourn and Woods [18] it was shown that an application of the instability described above leads to excellent agreement between observations of the differential emission measure and our theory for this quantity, which relates to the spectrum of frequencies of electromagnetic waves propagating out of the transition region. The agreement was good over the whole of the transition region temperature range, $10^{4}-10^{6} \mathrm{~K}$ with the expression for the heat flux given by

$$
q=-\kappa T^{\prime} \quad\left(\kappa=1.2 \times 10^{1.5}\left(T_{5}^{2.5}+g T_{5}^{-2.5}\right)\right)
$$

where $T_{5}=T / 10^{5}$ and $g \approx 1$ is a constant whose precise value is not given by the theory.
To a first approximation we may assume that $q$ is a constant, $-q_{0}$ say, and then

$$
T^{\prime}=\frac{q_{0}}{1.2 \times 10^{1.5}\left(T_{5}^{2.5}+g T_{5}^{-2.5}\right)} .
$$

Our theory does not yield a value for $q_{0}$, however Mariska [19] applied spectroscopic data to a cell-centred empirical model of the transition region to deduce $T^{\prime}$ as a function $\log T$. He found that the maximum value of the derivative occurs at $T=10^{5} \mathrm{~K}$ and has a value of $33 \mathrm{~K} \mathrm{~m}^{-1}$. Adopting this value in equation (8) we obtain $q_{0}=1.25 \times 10^{3}(1+g) \approx 2.5 \times 10^{3} \mathrm{~W} \mathrm{~m}^{2}$ and

$$
T^{\prime}=\frac{33(1+g)}{T_{5}^{2.5}+g T_{5}^{-2.5}} .
$$

In Figure 2 our theory for $T^{\prime}$ is compared with Mariska's empirical model for two values of $g$; the distribution is not sensitive to the value of this parameter and the agreement is good enough to allow us to assume that in equation (2) $q^{\prime} \approx 0$. (The dashed line in Fig. 2 has been calculated from the full energy equation, as will be described in Sect. 3.)

For the radiation term in equation (2) we shall assume that the transition region plasma (mainly hydrogen and helium) is singly and fully ionised. Thus if $n_{p}$ is the number density of the radiating elements, a radiation loss function $Q(T)$ is defined by

$$
\begin{aligned}
\mathcal{L}=n_{e} n_{p} Q(T)= & P^{2} Q(T) / T^{2}=10^{42} \wp^{2} Q(T) / T^{2} \\
& \left(\wp \equiv 10^{-21} n_{e} T\right) .
\end{aligned}
$$

For $Q(T)$ we shall adopt the function developed for solar coronal loop plasmas by Kankelborg and Longcope [20], a convenient approximation to which is given by the continuous curve with the polynomial form:

$$
Q(T)=10^{-d} \quad\left(z \equiv \log T_{5}=\log T-5\right),
$$

where

$$
\begin{aligned}
d= & 0.626 z^{7}-2.457 z^{6}+1.110 z^{5}+4.375 z^{4} \\
& -3.610 z^{3}-1.397 z^{2}+0.633 z+34.379 .
\end{aligned}
$$

For comparison we have also shown McClymont and Canfield's [21] piece-wise linear approximation

$Q(T)=10^{-34} T_{5}^{3}$ if $T_{5}<1, \quad Q(T)=10^{-34} / T_{5}$ if $T_{5}>1$.

From equation (10) we have

$$
\mathcal{L}=\wp^{2} 10^{37-d} / T T_{5}
$$

There remains the convective term $(\varrho u v)^{\prime}$ in equation (2). Now using the equation of state we may write $\varrho u=\frac{3}{2} p=$ $3 k_{B} n_{e} T=\epsilon \wp$, where $\epsilon=4.14 \times 10^{-2}$ is a dimensionless quantity. Hence this energy equation can be written

$$
\epsilon \frac{\partial \wp}{\partial t}+\epsilon(\wp v)^{\prime}=-q^{\prime}+\mathcal{H}-\mathcal{L},
$$

which is now susceptible to stability analysis. 


\section{Application of the energy equation}

Equation (13) governs the evolution of the pressure $\wp$. This equation is highly nonlinear with the pressure $\wp$ appearing as a factor in each term except the thermal conductivity $q^{\prime}$, which depends only on the temperature. In order to investigate the stability of the magnetic flux tubes, we perturb the system about the steady state $\wp_{0}$ by writing $\wp(t)=\wp_{0}+\wp_{1}(t)$, where $\wp_{1}(t)$ is a small change in $\wp$ at constant temperature. We shall ignore both the product $\wp_{1}(t)^{2}$ and the gradient $\wp^{\prime}$, which is a very small number depending on $\varrho g$, where $g$ is the gravitational acceleration. It follows from equations (6), (12) and (13) that

$$
\begin{aligned}
\frac{\partial \wp_{1}}{\partial t} & = \\
\quad & \left\{-v^{\prime}+\frac{5.55 \times 10^{-4} B_{g}}{T_{5}^{2} r}-2 \frac{2.42 \times 10^{-4} \wp_{0} 10^{37-d}}{T_{5}^{2}}\right\} \wp_{1}
\end{aligned}
$$

where $B_{g}$ is the magnetic field expressed in gauss and $r=10^{-4} R$. It is convenient to consider this equation in the form

$$
\frac{\partial \wp_{1}}{\partial t}=\left(A-B \wp_{0}\right) \wp_{1}
$$

where the values of $A$ and $B$ follow from equation (14). We can then draw the following conclusions about the behaviour of the model solutions:

(a) For any temperature $A>B \wp_{0}$, the system is locally unstable since any small perturbations to the pressure will grow exponentially in time.

(b) Since $A$ and $B$ have different temperature dependencies, stability over the whole of the transition layer does not exist; the best we can achieve is an average stability

$$
\langle A\rangle \leq\langle B\rangle \wp_{0}
$$

where $\langle\ldots\rangle$ denotes the average taken over the transition layer. Under these conditions the solutions are constant or small perturbations will decay in time, restoring stability to the system.

(c) Assuming that equation (16) applies, it follows from Figure 3 that there will exist unstable regions outside the temperature range $10^{4.5}-10^{6.2} \mathrm{~K}$, where the heating rate will exceed the radiation rate, i.e. where $\langle A\rangle>\langle B\rangle \wp_{0}$ and a pressure instability is generated.

(d) Finally, the instability cannot persist for long periods of time since a local increase in $\wp$ increases the radiation rate which will momentarily restore stability. However we cannot expect the pressure to adjust to its equilibrium value at each point in the transition layer as this would require large and persistent variations in the pressure with height, which would be quickly eliminated by sound waves.

This instability is the mechanism which we believe to be the cause of blinkers. In Figure 4 we have reproduced the downward velocity distribution obtained by Bewsher

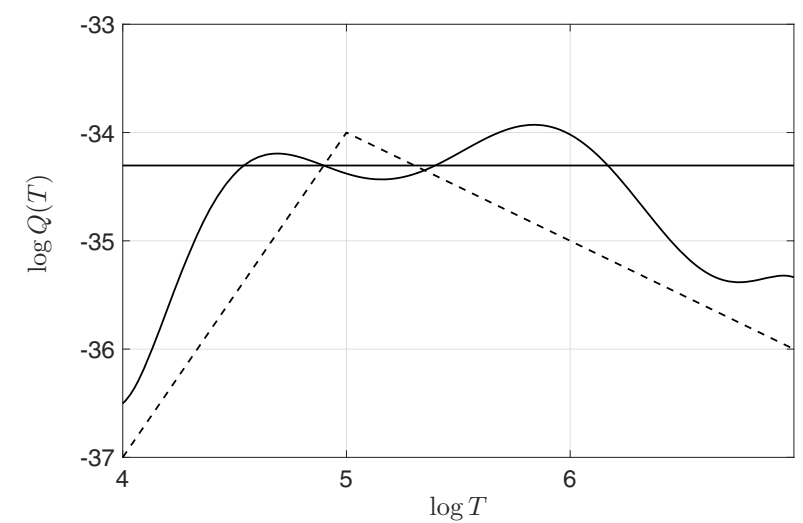

Fig. 3. The radiative loss function $Q(T)\left(\mathrm{erg} \mathrm{cm}^{3} \mathrm{~s}^{-1}\right)$ plotted for different temperature values throughout the transition region. The solid line shows the value of this function predicted by the model described in this paper and calculated using equation (11). The dashed line shows the values obtained by the piece-wise linear approximation used by McClymont and Canfield [21]. The horizontal line shows the first intersection of these two functions.

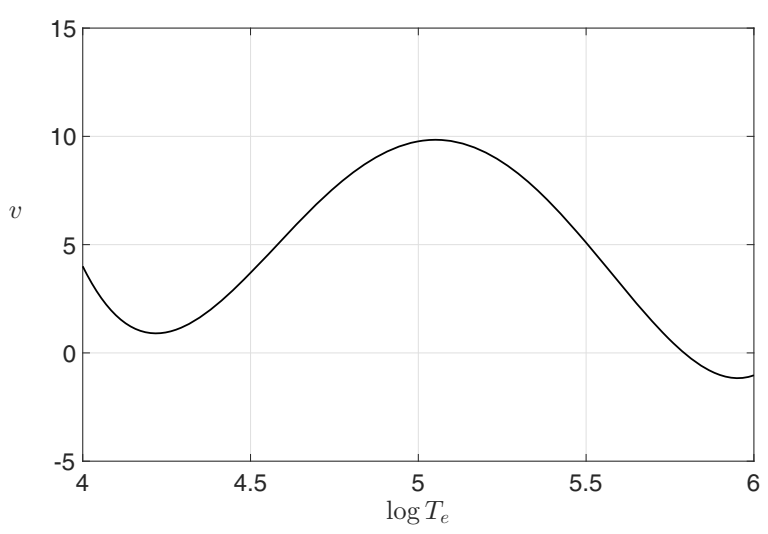

Fig. 4. Plots of observed Doppler velocities $\left(\mathrm{km} \mathrm{s}^{-1}\right)$ in the solar transition region. This plot is a reproduction of the data obtained by Bewsher et al. [4] who applied fourth-order polynomial fits to datasets from other studies.

et al. [4] from observational data. From this figure we notice that the gradient $v^{\prime}$ is negative for $10^{4} \mathrm{~K}<T<$ $10^{5.4} \mathrm{~K}$ and is therefore destabilizing in this temperature regime. With the help of equation (9) in this range we obtain the estimate $-v^{\prime}=2.9 /\left(T_{5}^{2.5}+T_{5}^{-2.5}\right)$, which is to be compared with the value $\sim 0.5 \wp_{0} / T_{5}^{2}$ of the radiation term. Since $\wp_{0} \sim 1$, we can conclude that at $T=10^{5} \mathrm{~K}$ the transition layer is unstable, as the destabilizing velocity term is much greater than the radiation term, and that the large pressure increase required to stabilise this point is unlikely to be achieved.

This conclusion could be incorrect for the following reason - specifically it may not be the case that the behaviour is dominated by the velocity term, since we have not allowed for the existence of a small volume filling factor, $\mathcal{F}$ say. The pressure in the threads would then be proportional to $\wp / \mathcal{F}$ and the radiation losses would be increased to $\mathcal{L} / \mathcal{F}^{2}$ per unit volume of thread or $\mathcal{L} / \mathcal{F}$ per unit 
volume of plasma loop. If this volume filling factor were present, then this same term would appear as a multiplicative factor in the second right-hand term in equation (14) and if $\mathcal{F} \approx 0.005$, say, we could certainly ignore the $v^{\prime}$ term in equation (14) as this term would then be negligible in comparison to the other terms. There is also the possibility that the observed Doppler velocities only apply to the plasma between the threads.

Pursuing this case, instead of equation (14) we obtain the equation

$$
\frac{\partial \wp_{1}}{\partial t}=\left\{5.55 \times 10^{-4} B_{g} / r-4.84 \times 10^{-4} \wp_{0} 10^{37-d}\right\} \wp_{1} / T_{5}^{2} .
$$

From equation (11) we find that the average of $10^{37-d}$ over the transition region temperature range $10^{4}-10^{6} \mathrm{~K}$ is $5.25 \times 10^{2}$ and therefore equilibrium with this average rate of energy loss requires that $B_{g} / r=458 \mathrm{GK} \mathrm{m}^{-1}$ or $B_{g}=4.58 \times 10^{-2} R_{t h}$, where $R_{t h}$ is the radius of the thread and $B_{g}$ is the magnetic field inside an individual thread. In the active region, $B_{g}$ lies within the range 1000-2000 G (Mariska [19]). For example, if we suppose that $B_{g}=1500 \mathrm{G}$, then the average equilibrium condition yields $R_{t h}=33 \mathrm{~km}$. It is our hypothesis that the thread assumes a radius such that the ohmic heating is more or less balanced by the radiation losses. Blinkers have been observed in both the quiet region as reported by Bewsher et al. [22] and in the active region as noted by Parnell et al. [8]. Here we have chosen to use parameters taken from the active region, although using parameters from the quiet region is also feasible and leads to the same conclusions.

This hypothesised balancing process is stable. For example, from equation (17) we see that if the radius is too large for this balance, the right-hand side of the equation will be negative and the pressure will be reduced until the radiation losses come into balance with the heating rate or more likely, the tendency of the pressure to decrease will result in the compression of the thread until its small radius results in an average balance. It is clear from Figure 3 that a constant radius throughout the transition layer is unattainable due to the varying losses. We can describe how the radius should vary with temperature in the steady state by solving the steady state form of equation (17), namely

$$
B_{g} / r=0.872 \wp_{0} 10^{37-d}
$$

subject to the condition that the magnetic flux through the base of the thread is constant, i.e. that $\pi R^{2} B=\Phi=$ constant. Taking $\Phi$ to have the average values determined by the values of $B$ and $R$ obtained in the previous paragraph and recalling that $\wp=\mathcal{O}(1)$, we find that the solution of this equation is

$$
r=10^{4} R_{t h}=26.5 \times 10^{(d-37) / 3} .
$$

We have plotted this radius in Figure 5, indicating the average equilibrium radius at $r=3.3 \times 10^{4} \mathrm{~m}$ by a dashed line.

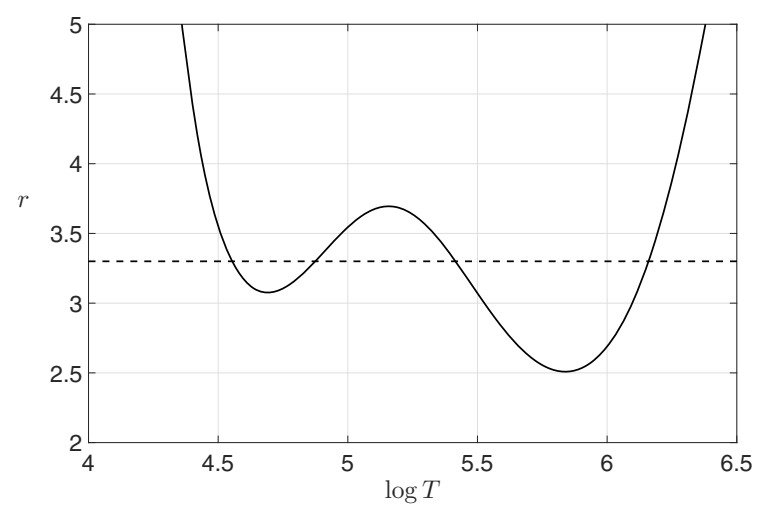

Fig. 5. The equilibrium radius in the magnetic thread $r$ $\left(10^{4} \mathrm{~m}\right)$, which has been plotted using equation (3). The dashed line shows a typical average equilibrium radius $(r=$ $\left.3.3 \times 10^{4} \mathrm{~m}\right)$.

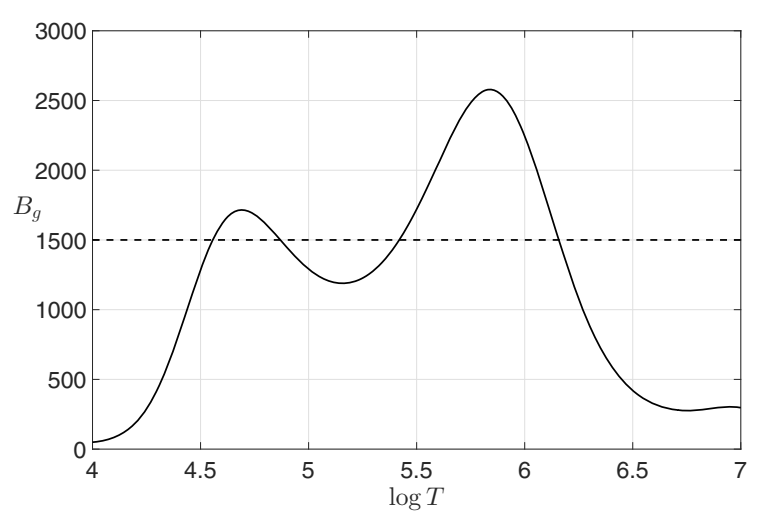

Fig. 6. The equilibrium magnetic field in the magnetic thread $B_{g}(\mathrm{G})$, which has been plotted using equation (18). The dashed line shows a typical average equilibrium magnetic field $\left(B_{g}=1500 \mathrm{G}\right)$.

In Figure 6 we have plotted the variation in the equilibrium magnetic field through the transition region. We do not expect this to be valid except in the region where the radiation losses are significant, but the main conclusion is that the magnetic field is relatively strong in this region. The large magnetic field in the transition region results in a large magnetic pressure in the flux thread.

From the argument above we have shown that the balance between the radiation term and the heating term, which is due solely to ohmic heating, is a stable process. That is, if a small perturbation is made to the thread in the transition region, then the pressure and radius of the thread will change to restore stability to the system. When this radiative balance is attained, the heating and radiation terms in equation (14) will approximately cancel and then the sign of the $v^{\prime}$ term will dominate the stability of the system. In particular, as described above the transition layer will be unstable in the range $10^{4} \mathrm{~K}<T<10^{5.4} \mathrm{~K}$. Hence the balance between the heating terms and radiation losses is a necessary condition for the transition region instabilities which we believe to be the cause of blinkers.

We propose that the mechanism which causes these instabilities is shock waves in the transition region. 
Observational data have revealed the presence of shocks in the solar transition region associated with photospheric magnetic flux reduction, e.g. Ryutova and Tarbell [23] were able to observe the signatures of shocks and their evolution from SUMER spectral data. These shocks are produced by colliding and reconnecting magnetic flux tubes, which release large amounts of energy in the form of MHD waves (e.g. see Harrison et al. [24]). Due to the strong stratification of the photosphere, those waves that propagate upwards in the direction of decreasing density quickly become shocks. When these propagating shocks pass through the magnetic flux tubes in the solar transition region, the local temperature is increased which in turn increases the magnitude of the plasma velocity, causing the instability to set in. The instability causes a rapid increase in the local pressure leading to a minor explosion and these explosions are observed as the small flare-like patches known as blinkers. The explosion dissipates energy back into the transition region, thus reducing the local pressure and eventually restoring stability.

\section{Model robustness}

It should be noted that the conclusions drawn above could simply be a manifestation of the specific transfer function chosen. We therefore investigate this possibility by using an alternative radiative transfer function and noting that the instability still exists.

Suppose that instead of the radiative transfer function in equation (11) used above, we instead adopt the usual power law relationship

$$
Q(t)=\alpha T^{\gamma}
$$

where we choose the constants $\alpha$ and $\gamma$ such that $\mathcal{L}=$ $2 \times 10^{-2} \mathrm{~W} \mathrm{~m}^{-3}$ at $T=10^{5} \mathrm{~K}$ and $\mathcal{L}=2 \times 10^{-5} \mathrm{~W} \mathrm{~m}^{-3}$ at $T=5 \times 10^{6} \mathrm{~K}$, which are the values for the canonical hot loop model (see Bray et al. [11]). We then find (see Ashbourn et al. [25]) that the appropriate radiation loss function is

$$
\begin{gathered}
\mathcal{L}=a P^{2} T^{\gamma-2}=10^{42} \wp^{2} a T^{\gamma-2} . \\
\left(a=1.43 \times 10^{-33}, \gamma \approx-0.306\right)
\end{gathered}
$$

Substituting into the energy equation we thus obtain

$$
\frac{\partial \wp_{1}}{\partial t}=\left\{-v^{\prime}+\frac{5.55 \times 10^{-4} B_{g}}{T_{5}^{2} r}-\frac{8.44 \times 10^{-3} \wp_{0} T_{5}^{\gamma}}{T_{5}^{2}}\right\} \wp_{1} .
$$

This is, as expected, of the same form as equation (14) and it thus follows immediately that the same conclusions about the stability of the transition layer (a)-(d) can be drawn by redefining our functions $A$ and $B$ as above. In this way, the same conclusions and hypotheses can be drawn for any model radiative loss function, which gives robustness to the results derived here.

\section{Conclusion}

In this paper we have presented a mathematical model of magnetic flux loops in the solar transition region in order to explain the phenomenon of blinkers. Modelling the dynamics of the transition region in this way allows far more detail about the physical processes in this region to be obtained than is possible with current observational instruments and techniques.

We have used an energy conservation equation applied to our model in order to obtain equations which govern the temperature gradients within the transition region. Our theory is shown to agree with experimental and empirical results in the literature. We have been able to find expressions for each of the terms in the energy equation and hence have used the model in order to obtain a partial differential equation governing the temporal evolution of the pressure within the transition layer magnetic flux tubes. Whilst this equation cannot be solved analytically, we have been able to perform a linear stability analysis to examine the stability of the transition layer for a range of different temperatures.

Based on the results of the model analysis we have been able to devise two hypotheses: namely that the major mechanism driving the formation of blinkers is the pressure instability inherent in the governing equations and that the magnetic threads assume a radius such that ohmic heating is balanced by the radiation losses. This radiative balance is an essential prerequisite for the pressure instability which we believe to be the cause of blinkers. We argue that this balancing process is stable and that whenever the pressure instability is switched on, the local pressure is reduced to bring the radiation losses into balance with the heating rate. This restores stability to the system on a timescale of a few minutes which is consistent with the reported observations of blinkers.

J.M.A.A. was supported by a Royal Commission for the Exhibition of 1851 Research Fellowship for part of this research.

\section{References}

1. R.A. Harrison, Sol. Phys. 175, 467 (1997)

2. T. Nishikawa, Sol. Phys. 105, 339 (1986)

3. G.W. Pneuman, R.A. Kopp, Sol. Phys. 57, 49 (1978)

4. D. Bewsher, C.E. Parnell, C.D. Pike, R.A. Harrison, Sol. Phys. 215, 217 (2003)

5. T. Tarbell, M. Ryutova, J. Covington, A. Fludra, Astrophys. J. 514, L47 (1999)

6. S. Subramanian, M.S. Madjarska, J.G. Doyle, D. Bewsher, Astron. \& Astrophys. 538, A10 (2012)

7. J. Chae, H.M. Wang, P.R. Goode, A. Fludra, U. Schuhle, Astrophys. J. 528, L119 (2000)

8. C.E. Parnell, D. Bewsher, R.A. Harrison, Sol. Phys. 206, $249(2002)$

9. S. Krucker, A.O. Benz, M.J. Aschwanden, Astron. \& Astrophys. 104, 99 (1997)

10. S. Subramanian, M.S. Madjarska, R.C. Maclean, J.G. Doyle, D. Bewsher, Astron. \& Astrophys. 488, 323 (2008) 
11. R.J. Bray, I.E. Cram, C.J. Durrant, R.E. Loughead, Plasma Loops in the Solar Corona (Cambridge University Press, 1991)

12. J.A. Klimchuck, Sol. Phys. 193, 53 (2000)

13. D.G. Wentzel, NASA SP-450 94, 100 (1981)

14. J.B. Zirker, Sol. Phys. 148, 43 (1993)

15. P. Brekke, Sol. Phys. 190, 379 (1999)

16. L.C. Woods, Thermodynamic Inequalities in Gases and Magnetoplasma (Wiley-Blackwell, 1996)

17. A.N. Krall, A.W. Trivelpiece, Principles of Plasma Physics, (McGraw-Hill, 1973)

18. J.M.A. Ashbourn, L.C. Woods, Phys. Rev. Lett. 84, 3748 (2000)

19. J.T. Mariska, The Solar Transition Region (Cambridge University Press, 1992)

20. C. Kankelborg, D. Longcope, Sol. Phys. 190, 59 (1999)
21. A.N. McClymont, R.C. Canfield, Astrophys. J. 256, 483 (1983)

22. D. Bewsher, C.E. Parnell, R.A. Harrison, Sol. Phys. 206, $23(2002)$

23. M. Ryutova, T. Tarbell, Phys. Rev. Lett. 541, L49 (2003)

24. R.A. Harrison, J. Lang, D.H. Brooks, D.E. Innes, Astron. \& Astrophys. 351, 1115 (1999)

25. J.M.A. Ashbourn, L.C. Woods, R. Bingham, Astrophys. J. 553, 941 (2001)

Open Access This is an open access article distributed under the terms of the Creative Commons Attribution License (http://creativecommons.org/licenses/by/4.0), which permits unrestricted use, distribution, and reproduction in any medium, provided the original work is properly cited. 